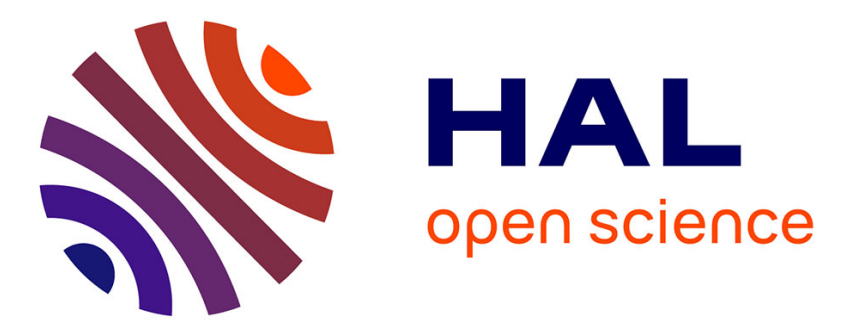

\title{
Segmentation of clustered cells in negative phase contrast images with integrated light intensity and cell shape information
}

Yuliang Wang, C. Wang, Zaicheng Zhang

\section{- To cite this version:}

Yuliang Wang, C. Wang, Zaicheng Zhang. Segmentation of clustered cells in negative phase contrast images with integrated light intensity and cell shape information. Journal of Microscopy, 2018, 270 (2), pp.188-199. 10.1111/jmi.12673 . hal-01808590

\section{HAL Id: hal-01808590 \\ https://hal.science/hal-01808590}

Submitted on 5 Jun 2018

HAL is a multi-disciplinary open access archive for the deposit and dissemination of scientific research documents, whether they are published or not. The documents may come from teaching and research institutions in France or abroad, or from public or private research centers.
L'archive ouverte pluridisciplinaire HAL, est destinée au dépôt et à la diffusion de documents scientifiques de niveau recherche, publiés ou non, émanant des établissements d'enseignement et de recherche français ou étrangers, des laboratoires publics ou privés.

\section{(ㅇ)(1) 80}

Distributed under a Creative Commons Attribution - NonCommercial - ShareAlikel 4.0 


\title{
Segmentation of clustered cells in negative phase contrast images with integrated light intensity and cell shape information
}

\author{
Y. WANG* (D), C. WANG* \& Z. ZHANG $\dagger$ \\ *School of Mechanical Engineering and Automation, Robotics Institute, Beihang University, Beijing, China \\ †Université de Bordeaux \& CNRS, LOMA, Talence, France
}

Key words. Cell segmentation, clustered cells, grey-weighted distance transform, light intensity, negative phase contrast images, shape.

\section{Summary}

Automated cell segmentation plays a key role in characterisations of cell behaviours for both biology research and clinical practices. Currently, the segmentation of clustered cells still remains as a challenge and is the main reason for false segmentation. In this study, the emphasis was put on the segmentation of clustered cells in negative phase contrast images. A new method was proposed to combine both light intensity and cell shape information through the construction of grey-weighted distance transform (GWDT) within preliminarily segmented areas. With the constructed GWDT, the clustered cells can be detected and then separated with a modified region skeletonbased method. Moreover, a contour expansion operation was applied to get optimised detection of cell boundaries. In this paper, the working principle and detailed procedure of the proposed method are described, followed by the evaluation of the method on clustered cell segmentation. Results show that the proposed method achieves an improved performance in clustered cell segmentation compared with other methods, with $85.8 \%$ and $97.16 \%$ accuracy rate for clustered cells and all cells, respectively.

\section{Introduction}

Observation and analysis of live cells play a vital role in characterisation of cell behaviours for both biology research and clinical practices, including wound healing, immunoreaction, and cancer cell metastasis (Piccinini et al., 2013; Das et al., 2015). In these applications, individual cells are segmented in optical images to implement cell morphological analysis, cell classification and even tracking (Xing \& Yang, 2016). Cell image segmentation is a process of separating individual cells from background in images containing cells. This could be done

Correspondence to: Yuliang Wang, School of Mechanical Engineering and Automation, Robotics Institute, Beihang University, 37 Xueyuan Rd., Haidian District, Beijing 100191, China. Tel: +86-18612525756; fax: +86-10-82314554; e-mail: wangyuliang@buaa.edu.cn manually, which is tedious and time consuming. Therefore, automated cell image segmentation techniques become important for high throughout cell morphological and dynamics studies (Irshad et al., 2014; Wang et al., 2014).

Different methods have been proposed to implement automated cell image segmentation, including thresholding method (Chalfoun et al., 2013), morphological erosion method (Chowdhury et al., 2013), watershed method (Lin et al., 2003; Wahlby et al., 2004; Yi et al., 2013; Kim et al., 2014), level set method (Ersoy et al., 2008) and active contour models (Chenyang \& Prince, 1998; Cosatto et al., 2008; Ali \& Madabhushi, 2012; Seroussi et al., 2012; Vink et al., 2013), or the combination of several different algorithms. Most methods can achieve automated cell segmentation to some extent, especially for individual cells. However, the segmentation of clustered cells still remains as a challenge.

The methods used to segment clustered cells can be categorised into shaped-based (Kumar et al., 2006; Al-Kofahi et al., 2010; Kong et al., 2011; Wienert et al., 2012; Veta et al., 2013; Chen et al., 2015; Song et al., 2015; Gharipour \& Liew, 2016) and light intensity-based approaches (Ali et al., 2008; Wang et al., 2015). Shape-based approaches use distance transform (Al-Kofahi et al., 2010; Chen et al., 2015; Song et al., 2015), concave points along contours (Kumar et al., 2006; Wienert et al., 2012; Gharipour \& Liew, 2016), and radial symmetry transform (Kong et al., 2011; Veta et al., 2013; Gharipour \& Liew, 2016) to conduct cell segmentation, assuming cells have regular (elliptical, for example) and convex shapes. Light intensity-based methods segment cells using the special distribution of light intensity over cell bodies, assuming the light intensity has direct correlation with cell height. The commonly used methods are level set method (Ali et al., 2008) or peak detection method (Wang et al., 2015).

Both shape-based and light intensity-based methods have pros and cons in clustered cell segmentation. On one hand, light intensity, like phase contrast images, represents height information of cells. As a result, the light intensity information is useful in determining cell locations and exact boundaries 
between two contacting cells within an area of clustered ones. On the contrary, the shape-based methods only use the boundary information, which is normally obtained from a binarisation operation. This inevitably loses some useful information of the clustered cells. On the other hand, the light intensitybased methods require high image contrast. However, the actual cell images, including phase contrast images, suffer from various noises. The methods may lead to oversegmentation or undersegmentation. From above analysis, one can see that light intensity and cell shape information should be integrated to perform a more efficient segmentation, rather than being utilised solely.

Some efforts have been put to combine the shape and light intensity information (Lin et al., 2003; Wahlby et al., 2004; Ali \& Madabhushi, 2012; Dewan et al., 2014). These methods normally rely on some assumptions, either from shape or from light intensity point of view. For example, in the gradientweighted distance transform method, it is assumed that internuclei gradients are higher than intranuclei gradients (Lin et al., 2003). In the synergistic boundary and region-based active contour model, nuclei are assumed to be roughly elliptical in shape (Ali \& Madabhushi, 2012). The assumptions limit their applications and reduce their robustness.

Aiming at developing a clustered cell segmentation method coping with the complexity of live cells either on shape or light intensity in negative phase contrast images, we focus on fusing the light intensity and cell shape information. It gives an improved performance for clustered cell segmentation. Specifically, the fusion of the two information solves two issues existed in negative phase contrast image segmentation. One is the pseudo light intensity peaks caused by intracellular organelles, which could cause oversegmentation. The other is the reversed image contrast for cells with larger height (like cells under division), which could cause undersegmentation.

The rest of the paper is organised as follows. In the Experimental section, experimental details is introduced, including cell culturing, cell imaging and programming software. In the section of Principle of the clustered cell segmentation, the detailed process of clustered cell detection and separation are presented. In the Results and discussion section, the clustered cell segmentation using the proposed method is demonstrated, followed by the comparison and evaluation of clustered cell segmentation among different methods.

\section{Experimental}

In this study, an established nontumorigenic breast epithelial cells MCF 10A (Michigan Cancer Foundation 10A) were cultured for the experiments. The cells were maintained in 47.5\% Dulbecco's modified Eagle's medium and 47.5\% F-12 medium supplemented with 5\% horse serum, EGF (Epidermal Growth Factor) (20 ng mL $\left.\mathrm{m}^{-1}\right)$, hydrocortisone $\left(0.5 \mu \mathrm{g} \mathrm{mL}^{-1}\right)$, Cholora toxin $\left(0.1 \mu \mathrm{g} \mathrm{mL}^{-1}\right), \mathrm{NaHCO}_{3}(0.2 \mathrm{nM})$, and $1 \%$ penicillin/streptomycin.
Negative phase contrast images were acquired using a phase contrast microscope (IX71, Olympus, Japan) equipped with a $10 \times$ negative phase contrast lens. The image size is $1344 \times$ 1024 , corresponding to the actual field of view of $900 \mu \mathrm{m} \times$ $686 \mu \mathrm{m}$.

All algorithms developed in this study, including preprocessing, suspected clustered cell detection and skeleton-based clustered cell separation, were implemented in Matlab (Version 2016b, The Mathworks, Inc., USA).

\section{The principle of the clustered cell segmentation}

In this study, the shape and light intensity information are integrated to segment the clustered cells. The schematic of the segmentation is shown in Figure 1. For a simulated image shown in Figure 1(A), the preliminary cell shape information is obtained by thresholding operation to the cell image. Then a binarised image which indicates the preliminary shape of cells is obtained, as shown in Figure 1(B). The boundary of the mask can then be used to represent cell shape. Meanwhile, light intensity of cell bodies within the preliminarily segmented mask area can also be extracted (Fig. 1C). After that, the light intensity information and distance transform information are fused (Fig. 1D).

For the sake of the clustered cell separation, a skeletonbased separation algorithm is employed. The Skeleton (green line in Fig. 1E) of the preliminarily segmented area represents the distribution of cells in the region. The mask area can then be separated with assistant of the graph theory. The final step is the refinement of cell boundary detection using a contour expansion method (Wang et al., 2015), as shown in Figure 1(F).

\section{Results and discussion}

In this section, the detailed procedure of the proposed method is first demonstrated, followed by the evaluation of the proposed method.

\section{Construction of grey-weighted distance transform}

In this study, the grey-weighted distance transform (GWDT) (Soille, 1994) was operated on the raw images to integrate cell shape and light intensity information. For the preparation of GWDT, cell shape information was first extracted. Instead of using the thresholding method with a global fixed threshold value, here the adaptive binarisation method was applied (Roth \& Gerhard, 2007). A raw cell image and corresponding result after adaptive binarisation are shown in Figures 2(A) and (B), respectively. After that, the obtained binary images were processed by morphological close operation to remove the burrs near cell borders. The areas of less than 200 pixels were filtered out. The areas selected by rectangles in Figure 2(A) are two examples of the clustered cells. 


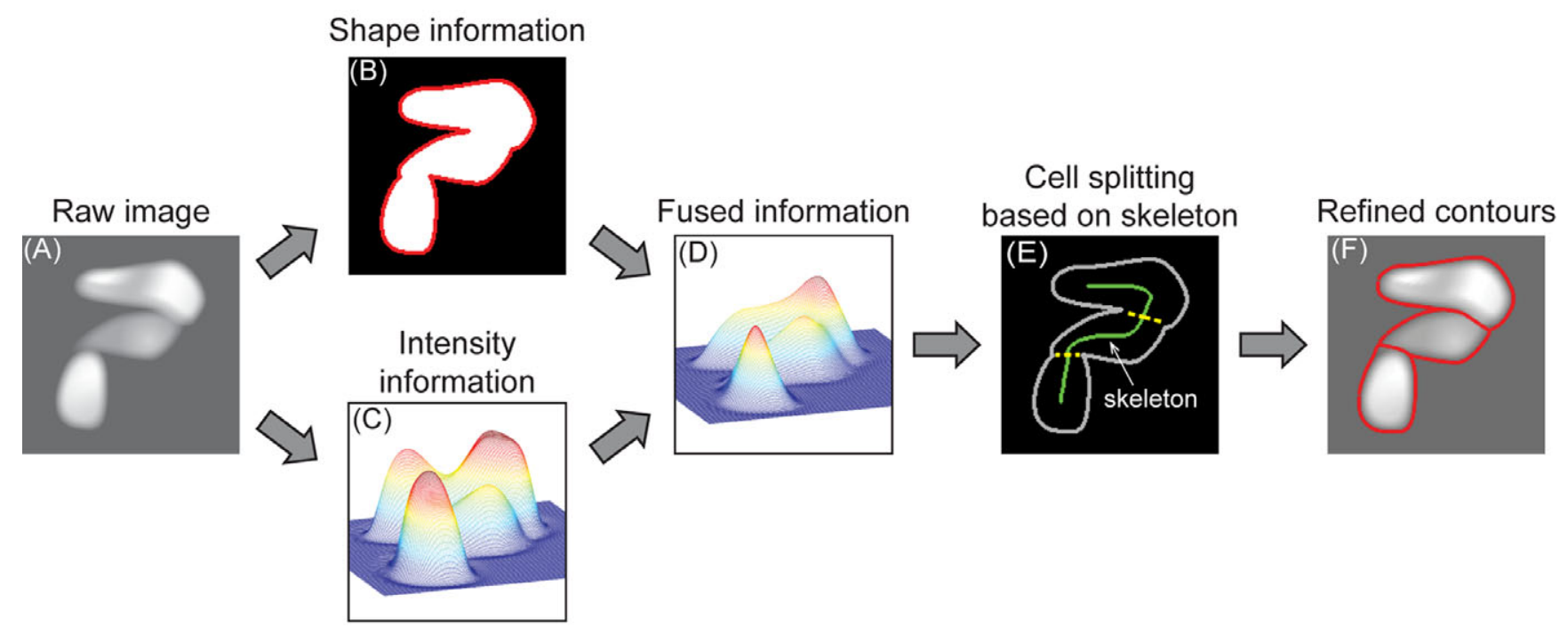

Fig. 1. Schematic diagram of the proposed segmentation scheme. (A) A simulated image showing three contacting cells in an area. (B) The binary image of (A), indicating the preliminary shape of the clustered cell area. (C) Mesh plot of light intensity in the area, where a spurious peak is detected. (D) Mesh plot of the grey-weighted distance transform by integrating shape and light intensity information, where the spurious peak is eliminated. (E) The skeleton of the clustered cell region and corresponding cell separation result. (F) Refined contours are obtained with an active contour method.
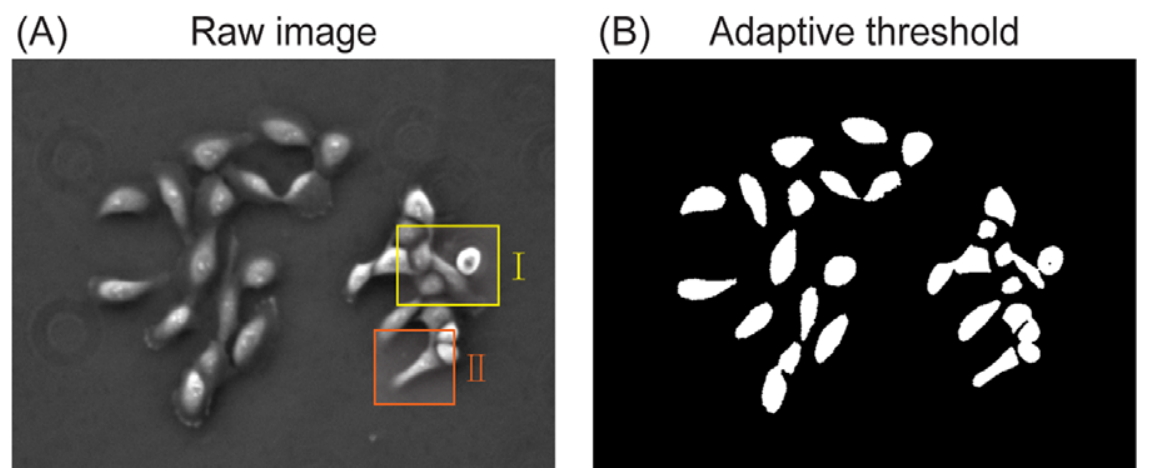

Fig. 2. Adaptive binarisation of cell images. (A) Raw negative phase contrast cell image. (B) Binary image with the adaptive threshold method. Cell shape information is obtained by the preliminary segmentation.

The detailed process of GWDT construction is demonstrated as follows. In a grey scale image $f$, take a path $P$ going from pixel $p_{0}$ in a foreground area to pixel $p_{m}$ in a background area as a $m$ tuple $\left(p_{0}, p_{1}, \ldots, p_{m}\right)$. The time $t_{f}(P)$ is the accumulation of the mean intensity for any two neighbouring pixels along the path $P$, given by:

$$
\begin{aligned}
t_{f}(P) & =\sum_{i=1}^{m} \frac{f\left(p_{i-1}\right)+f\left(p_{i}\right)}{2} \\
& =\frac{f\left(p_{0}\right)}{2}+\frac{f\left(p_{m}\right)}{2}+\sum_{i=1}^{m-1} f\left(p_{i}\right) .
\end{aligned}
$$

The geodesic time $t_{f}\left(p_{0}, p_{m}\right)$ between $p_{0}$ and $p_{m}$ is defined as the smallest value of time for all the possible paths from pixel $p_{0}$ to pixel $p_{m}$, given as

$$
t_{f}\left(p_{0}, p_{m}\right)=\min \left\{t_{f}(P) \mid P \text { links } p_{0} \text { to } p_{m}\right\} .
$$

With the definition of geodesic time, the value $g\left(p_{0}\right)$ at pixel $p_{0}$ in a foreground area to the background $Y$ is defined as the smallest value of geodesic time linking $p_{0}$ to any pixel $y$ in $Y$, given as

$$
g\left(p_{0}\right)=t_{f}\left(p_{0}, Y\right)=\min _{y \in Y} t_{f}\left(p_{0}, y\right) .
$$

For three clustered cells shown in Figure 3(A), the cell shape information was first extracted through the thresholding method. A mask area containing the three clustered cells was obtained, as shown in Figure 3(B). Within the mask area, the light intensity can also be extracted (Fig. 3C). The GWDT can then be obtained using Eq. (3), as shown in Figure 3(D).

\section{Clustered cells detection}

Before the operation of clustered cell separation, one needs to determine clustered cells. In raw negative phase contrast 
(A)

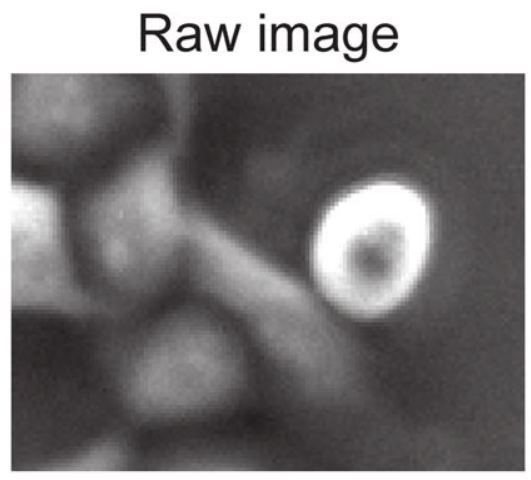

(C)

Intensity information

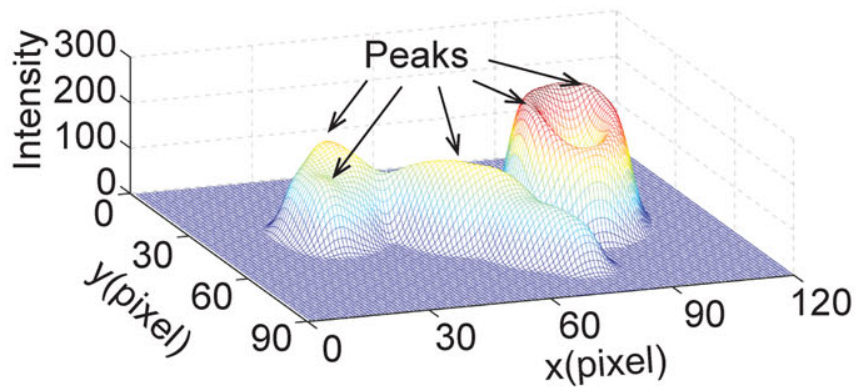

(B)

\section{Shape information}

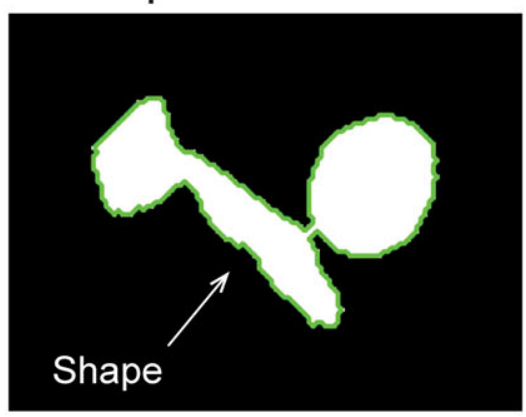

(D)

Fused information

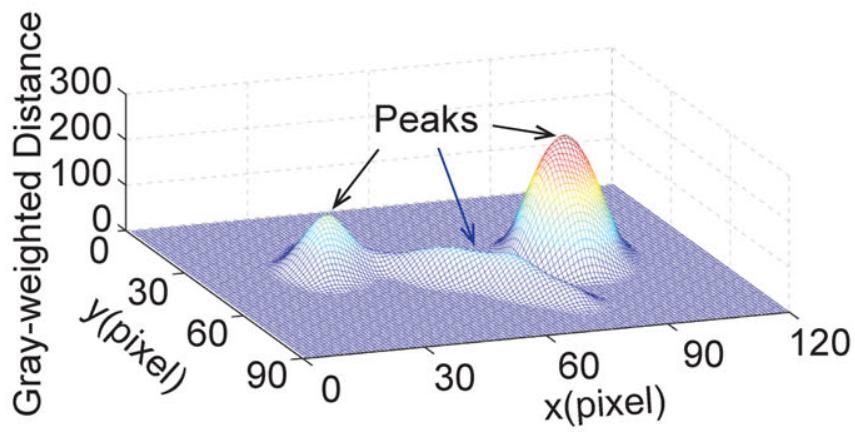

Fig. 3. Demonstration of the construction of grey-weighted distance transform. (A) A raw image of clustered cells. (B) Binary image of the clustered cells with a preliminarily detected mask area used to represent cell shape information. (C) Light intensity within the preliminarily detected mask area for the three clustered cells. Within the areas, two pseudo-peaks were detected, which normally cause oversegmentation. (D) Grey-weighted distance transform obtained by integrating cell shape and light intensity information. With the method, peaks can be correctly detected for each cell.

images, the light intensity achieves its higher value around nucleus area and gradually decreases towards cell boundaries. Therefore, cell locations can be determined by detecting the maximum value (peaks) (Wang et al., 2015). However, due to the existence of cell organelles, which have higher light refractive index and hence cause higher light intensity in cell images, the pseudo-peaks may be detected if only light intensity is considered, as shown in Figure 3(C). This will lead to oversegmentation.

In this study, instead of using light intensity, the GWDT was applied to detect clustered cells through peak detection. The regional maximum points were detected and taken as peaks. The peaks were then used to represent locations for individual cells. For the clustered cells shown in Figure 3(A), three peaks were detected with GWDT, as shown in Figure 3(D). One can see that the integration of shape and light intensity information helps to reduce oversegmentation.

The detection of clustered cells is demonstrated in Figure 4. For a raw image shown in Figure 4(A), the thresholding method was first applied to get the preliminary segmentation. The GWDT was then constructed in the preliminarily detected mask areas. The mask areas of clustered cells were detected through peak detection, as shown in Figure 4(B).
Masks containing only one peak were taken as individual cell regions, whereas the ones containing multiple peaks were taken as clustered cell regions. In the figure, the yellow and green mask areas correspond to the detected individual and clustered cells, respectively.

\section{Region skeleton-based clustered cell separation}

After clustered cell detection, the next step is to perform separation of them. Here a region skeleton-based method was proposed to separate clustered cells, including skeleton extraction, skeleton thinning, graph model representation, splitting points searching, dividing path searching and contour expansion.

A region skeleton is a curved line going through a mask area and normally has the equal distance to the boundary of the mask area. It is used to represent the topological features of clustered cells. In this study, augmented fast marching method (AFMM) (Telea \& Van Wijk, 2002) was used to extract skeletons in the mask areas. The method relies on the evolution of the parameterised boundaries of the mask areas. In the method, pixels along a boundary of a mask area are first coded to be parameterised. The parameterised boundary 

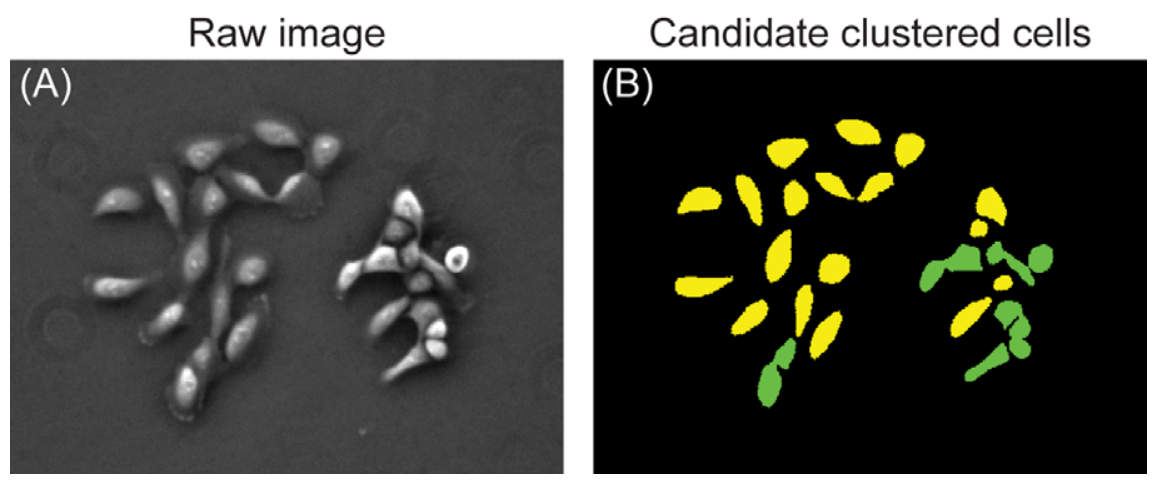

Fig. 4. Detection of the clustered cells. (A) A raw negative phase contrast image. (B) Result of clustered cell detection in the grey-weighted distance map. Green mask areas refer to suspected clustered cells whereas yellow areas refer to individual cells.

pixels are then evolved inward the mask area under a velocity field constructed by solving an Eikonal equation. When two pixels in the evolving boundary encounter each other and the difference of their codes is larger than a threshold value, the encountered pixels are transformed into a skeleton point. By doing so, the parameterised boundary will be converted into a skeleton for the mask area.

Take the clustered cells shown in Figure 3(A) and the preliminary segmentation shown in Figure 3(B) as examples. For the mask area, the skeleton was first obtained using the AFMM method, as shown in Figure 5(A). The skeletons directly obtained by AFMM are normally not single-pixel width, as shown in the inset enlarged figure. Here a modified thinning algorithm (Zhang \& Suen, 1984; Chin et al., 1987; Holt et al., 1987) was used to transform the preliminarily obtained skeletons into 8-connected components. In the thinning processing, each foreground pixel is compared with eight different 3 $\times 3$ templates (Fig. 5B). In the templates, $P$ is the centre of the templates, 0 and 1 are the pixels in the background and foreground, respectively, and X means the value can be either 0 or 1 . When a pixel in the preliminarily detected skeletons pixel is superimposed with the centre of a template and the eight neighbourhood pixels in the image are also matched with that of the template, the pixel will be deleted. The eight templates scan the foreground pixels in the image until no more foreground pixel can be deleted. The remaining skeleton will be taken as the final one for the mask area. Figure 5(C) shows the obtained skeleton after the thinning processing.

With the obtained skeletons, the graph theory is applied to better represent the relationship among different regions in the mask area. Figure 5(D) shows the diagrammatic form of the graph corresponding to the skeleton shown in Figure 5(C).

The graph is consisted of vertices and edges. The contiguous vertices are linked by edges. In the construction of graphs, the pixels in the extracted skeleton are divided into three types: end-points, junction-points and connecting-points (Bai \& Latecki, 2008). An end-point $\left(V_{1}, V_{2}\right.$, and $V_{3}$ in this case) is a pixel at the end of a skeleton branch with only one pixel from the skeleton in its eight neighbourhoods. A junction-point $\left(V_{4}\right)$ is the intersection of different skeleton branches, with at least three pixels in its eight neighbourhoods. All the rest pixels in the skeleton are defined as connecting-points. The end-points and junction-points are called vertices. The skeleton branches connecting any two vertices are defined as edges $\left(e_{1}, e_{2}\right.$ and $\left.e_{3}\right)$.

The constructed graphs can be represented by three elements, numbered vertex set $V=\left\{V_{1}, V_{2}, V_{3}\right\}$, edge set $E=$ $\left\{e_{1}, e_{2}, e_{3}\right\}$ and relations $\varphi$ among vertices and edges, which is given as $\varphi: E \rightarrow E(V)=\{(u, v) \mid u, v \in V\}$. In this study, a modified graph $G$ is proposed. In the graph, the values of the GWDT along the detected skeleton are taken as the fourth element. The modified model $G$ can then be expressed as

$$
G=(V, E, \varphi, \boldsymbol{W}) .
$$

In the equation, $\boldsymbol{W}=\left\{\boldsymbol{w}\left(e_{1}\right), \boldsymbol{w}\left(e_{2}\right), \boldsymbol{w}\left(e_{3}\right)\right\}$, where $\boldsymbol{w}\left(e_{i}\right)$ represents the distribution of GWDT along a skeleton branch linking two vertices:

$$
\boldsymbol{w}\left(e_{1}\right)=\left\{d\left(V_{1}\right), d\left(p_{1}\right), d\left(p_{2}\right), \ldots, d\left(p_{n}\right), d\left(V_{4}\right)\right\},
$$

where $\left\{p_{1}, p_{2}, \ldots, p_{n}\right\}$ are the connecting-points along the skeleton path, and $d\left(p_{i}\right)$ is the value of GWDT.

In order to suppress the noise of the region boundary, the width vector $W$ is smoothed by a local regression and a second order polynomial model. The local minimums of the smoothed width vector $\boldsymbol{w}\left(e_{i}\right)$ correspond to the turning points of the GWDT values along the skeleton $e_{i}$ and are likely to be the contacting points of cells. The distribution of GWDT values along the three edges $\left(e_{1}, e_{2}\right.$ and $\left.e_{3}\right)$ is shown in Figure 5(E). Two local minimums were found along the curves of $\boldsymbol{w}\left(e_{1}\right)$ and $w\left(e_{2}\right)$, corresponding to two splitting points in the cell region. Because cells have certain size, here the skeleton branches with length smaller than 10 pixels are ignored, like the red curve $\boldsymbol{w}\left(e_{3}\right)$ in Figure 5(E).

With the detected splitting points, the dividing paths were searched to split the clustered cells by searching slope-lines (Serrat et al., 2000). In hydrology, the water flow runs downhill along the steepest slope, which is called slope-lines. 
(A) Skeleton after AFMM

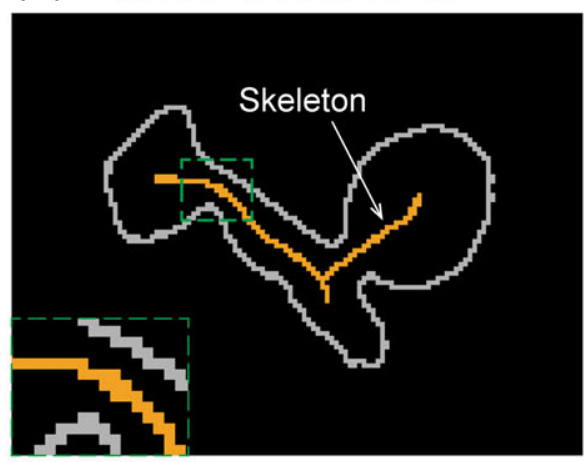

(C) Skeleton after thinning

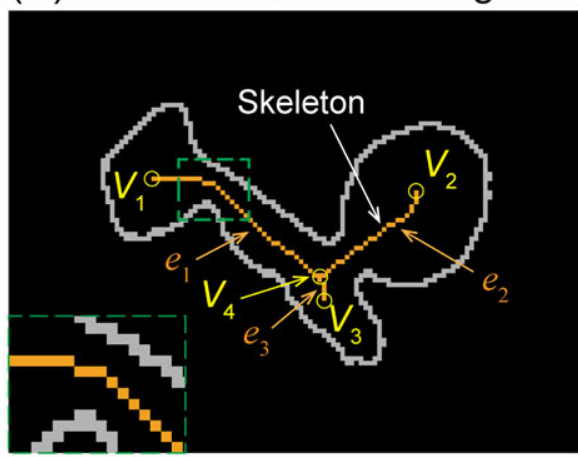

(F) Dividing paths in 3D

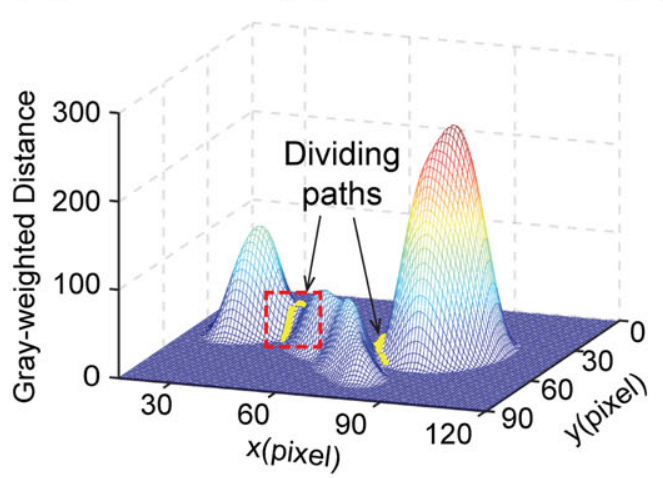

(B)

\begin{tabular}{|c|c|c|}
\hline 1 & $X$ & 0 \\
\hline 1 & $P$ & 0 \\
\hline 1 & $X$ & 0 \\
\hline
\end{tabular}

\begin{tabular}{|c|c|c|}
\hline$X$ & 1 & $X$ \\
\hline 1 & $P$ & 0 \\
\hline$X$ & 0 & 0 \\
\hline
\end{tabular}

(D)

Graph

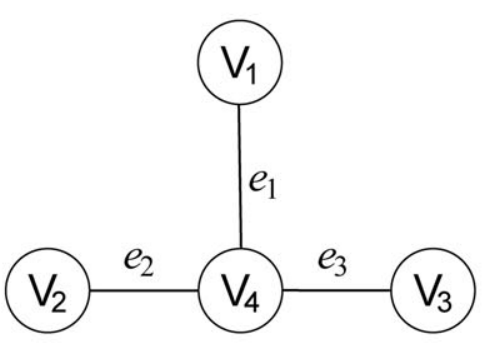

(G)

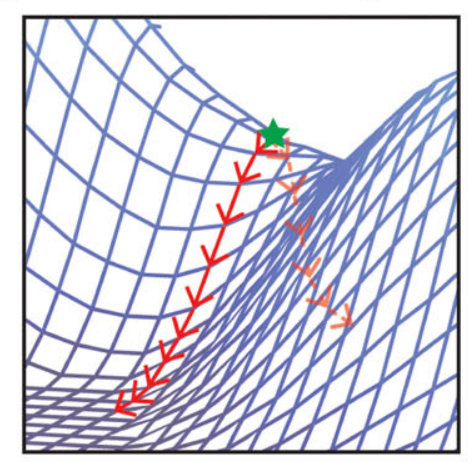

Thinning templates

\begin{tabular}{|c|c|c|}
\hline 1 & 1 & 1 \\
\hline$X$ & $P$ & $X$ \\
\hline 0 & 0 & 0 \\
\hline \hline$X$ & 1 & $X$ \\
\hline 0 & $P$ & 1 \\
\hline 0 & 0 & $X$ \\
\hline
\end{tabular}

(E)
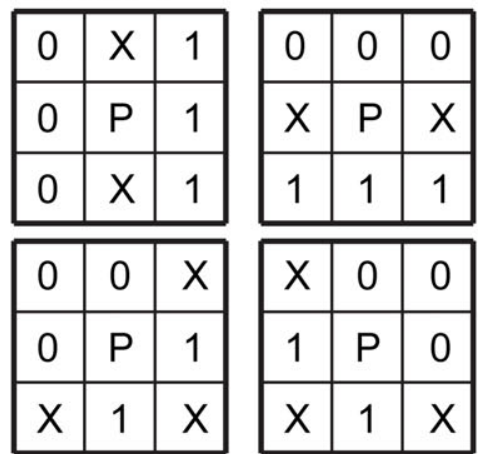

Width distribution curves

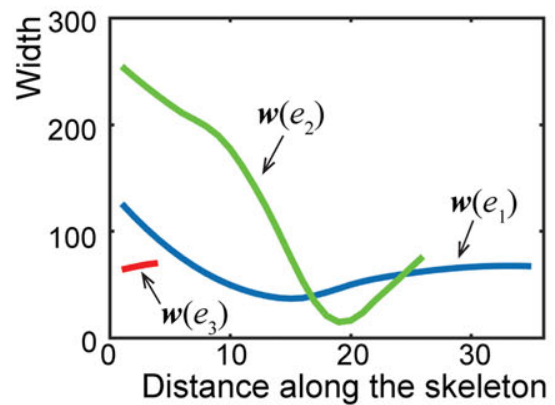

(H) Dividing paths in 2D

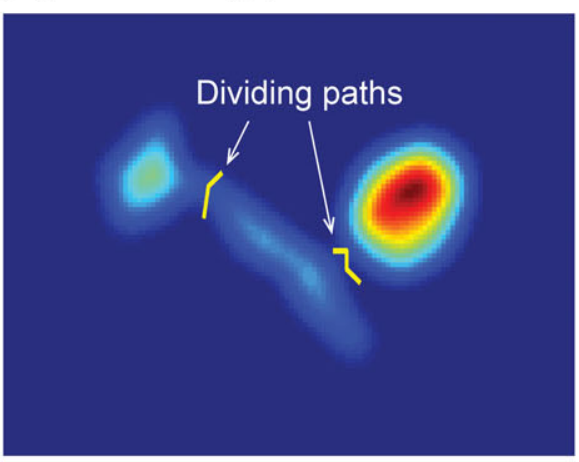

Fig. 5. Demonstration of the region skeleton-based clustered cell separation. (A) A skeleton of the mask area in Figure $3 b$ is obtained (orange curve) with AFMM method. The inset in the bottom left corner is the enlarged area of the green dashed box. (B) Templates for the skeleton thinning operation. In the templates, $P$ is the centre of the templates, 0 and 1 are the pixels in the background and foreground, respectively, and $\mathrm{X}$ means the pixel can be either 0 or 1 . (C) The skeleton after thinning operation. $V_{1}, V_{2}$ and $V_{3}$ are end-points of the skeleton, and $V_{4}$ is the node-point. The inset in the bottom left corner is the enlarged area of the green dashed box. (D) Graph diagram of (C), where vertices are linked by edges of $e_{1}, e_{2}$ and $e_{3}$. (E) Width distribution curves of lines $e_{1}, e_{2}$ and $e_{3}$. Two local minimums are detected on curves $\boldsymbol{w}\left(e_{1}\right)$ and $\boldsymbol{w}\left(e_{2}\right)$, whereas $\boldsymbol{w}\left(e_{3}\right)$ is ignored due to its short length. (F) Dividing paths are searched on the smoothed grey-weighted distance map. (G) Enlarged area selected by the dashed box in (F). The star mark represents the start pixel corresponding to the local minimum of $w\left(e_{1}\right)$ in $(\mathrm{E})$, and the points of the path are searched one by one, as shown by arrows. The right half of the curve represents the path behind the surface. (H) 2D plot of smoothed grey-weighted distance map and dividing paths. Clustered cells are separated by the skeleton-based method with the fused information of intensity and shape.

In morphology, slope-lines refer to the paths along which the light intensity gradient achieves its highest values along the path. Assuming that the GWDTs are mountains, slope-lines split the mountains and realizes the optimal separation of contacting cells. In this paper, slope-lines are determined by searching the pixels with minimal intensity level in their eight-neighbourhood. The path searching starts from the splitting points. Two opposite pixels in the 


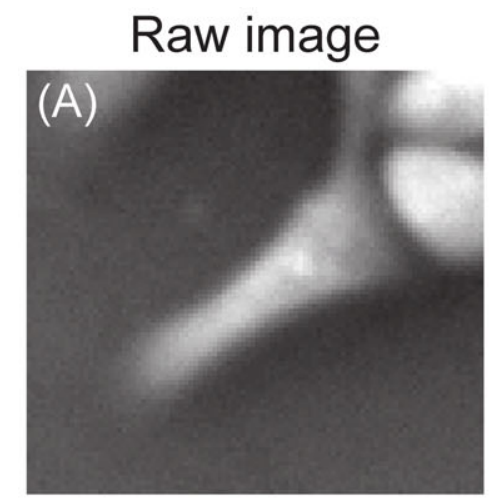

\section{Shape information}

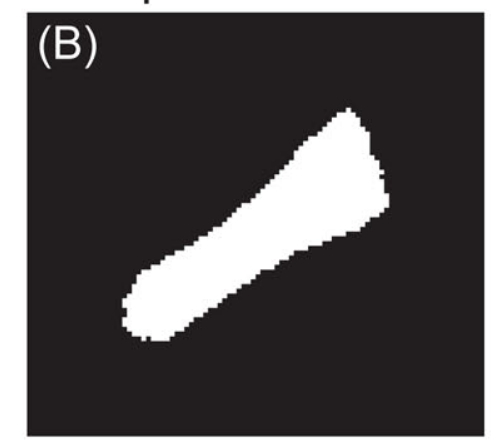

\section{Distance transform}

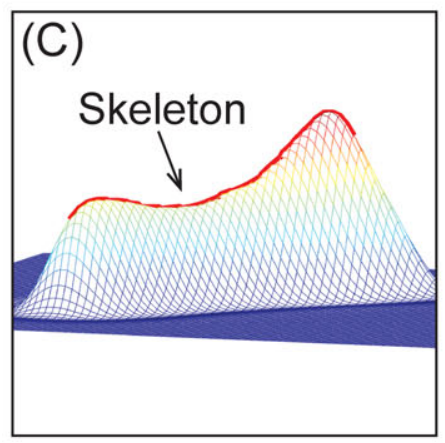

(D)

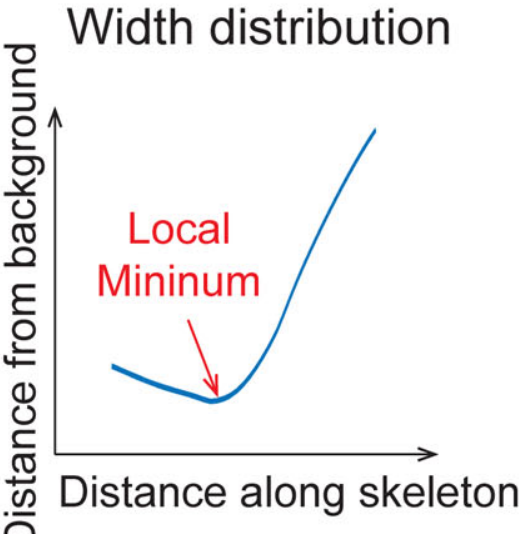

Cell contours

\section{Gray weighted} distance transform
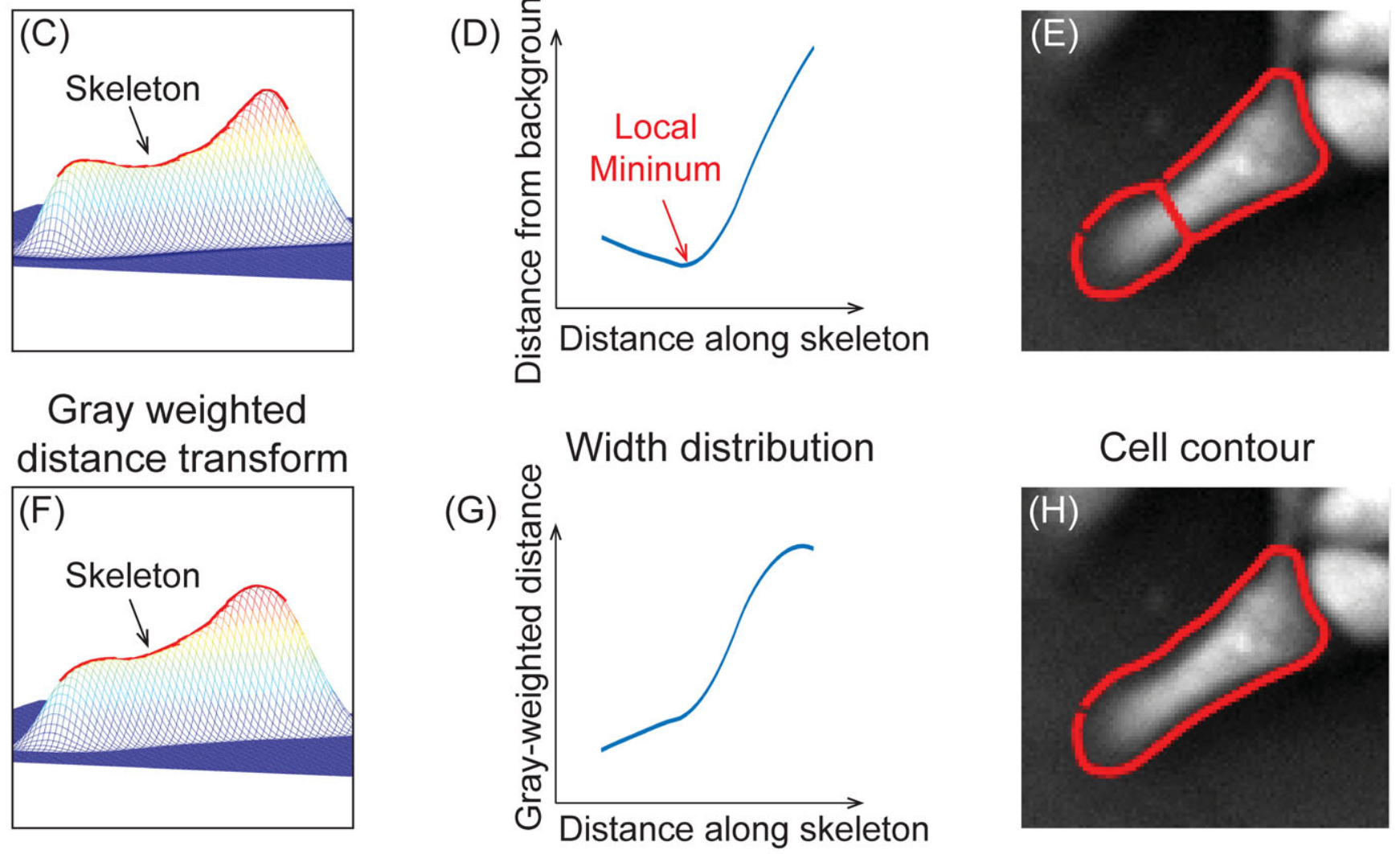

\section{Cell contour}

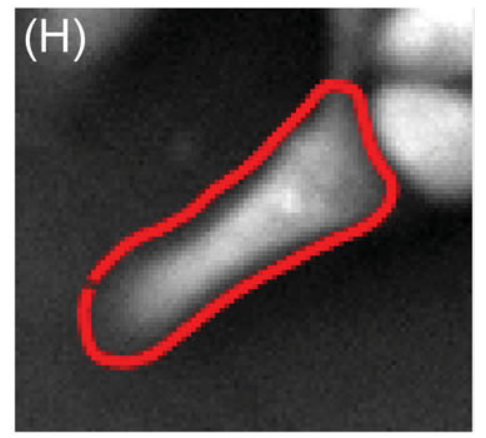

Fig. 6. Comparison of cell segmentation with grey-weighted distance transform and Euclidean distance transform. (A) Raw negative phase contrast image of an elongated cell. (B) Binary image of the elongated cell with only shape information. (C), (F) Mesh plot of Euclidean distance transform (C) and grey-weighted distance transform (F). (D), (G) Distribution of values along the skeleton in Euclidean distance transform (D) and grey-weighted distance transform (G), which corresponds to the red curves in (C) and (F). A local minimum was detected in (D). (E), (H) Segmentation results with distance transform and grey-weighted distance transform, respectively. The distance transform causes oversegmentation, whereas the grey-weighted distance transform gives a correct segmentation.

eight-neighbourhood which have smallest intensity value will be taken as the next point on the path, leading the directions of the path. Along the two directions, pixels with minimal intensity in eight-neighbourhood of current pixels will be added onto the path until it reaches the background, as shown in Figure 5(G). The separation results for the clustered cells in Figure 3(A) are shown in Figures 5(F) and (H) for 3D and 2D images, respectively.

Compared with the methods which solely rely on the shape or light intensity, the combination of GWDT and cell skeleton reduces oversegmentation and improves cell separation accuracy. Here we take a cell in Figure 6(A) as an example. The 

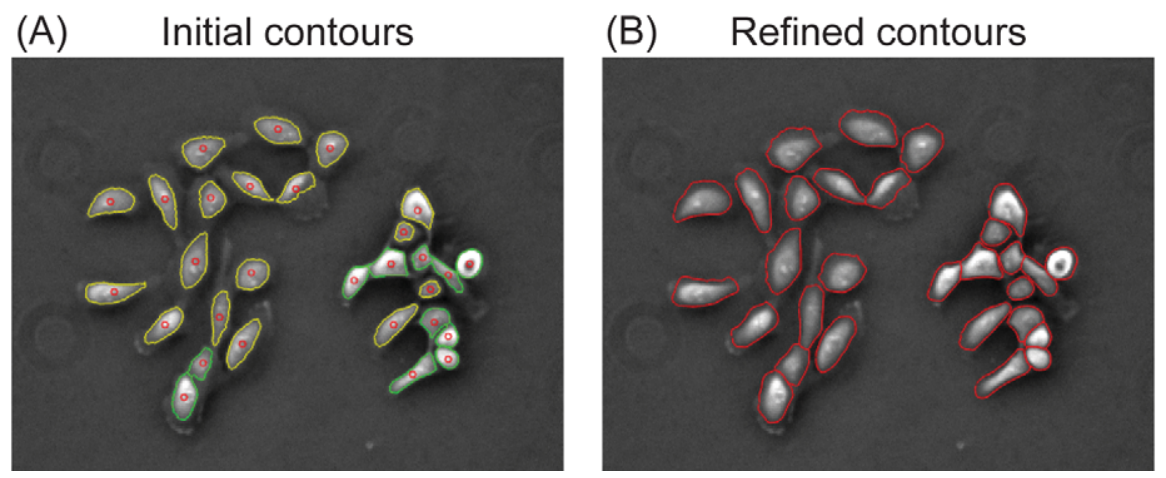

Fig. 7. Optimisation of cell boundary detection. (A) Initial contours extracted from preliminarily segmented mask areas. Yellow contours refer to the initial contours of individual cells, while the green ones refer to the initial contours of clustered cells after the skeleton-based separation method. (B) Result of the optimised contour detection after contour expansion operation.

Table 1. Comparison of clustered cell separation with four different methods.

\begin{tabular}{|c|c|c|c|c|c|c|c|}
\hline Methods & $\begin{array}{l}\text { Number of } \\
\text { cells }\end{array}$ & $\begin{array}{l}\text { Correct seg- } \\
\text { mentation }\end{array}$ & Oversegmentation & Undersegmentation & Accuracy rate & PPV & Recall \\
\hline $\begin{array}{l}\text { Watershed method (Meyer, } \\
\text { 1994) }\end{array}$ & & 279 & $93(23.7 \%)$ & $20(5.1 \%)$ & $71.0 \%$ & $75.00 \%$ & $93.31 \%$ \\
\hline $\begin{array}{l}\text { Intensity-based method } \\
\text { (Wang et al., 2015) }\end{array}$ & 393 & 315 & $37(9.4 \%)$ & $40(10.2 \%)$ & $80.2 \%$ & $89.49 \%$ & $88.73 \%$ \\
\hline The proposed method & & 337 & $24(6.1 \%)$ & $31(7.9 \%)$ & $85.8 \%$ & $93.35 \%$ & $91.58 \%$ \\
\hline
\end{tabular}

preliminarily detected mask area is shown in Figure 6(B). The mesh plot of the distance transform is shown in Figure $6(\mathrm{C})$. One can see that there is a local minimum of the width distribution along the detected skeleton (Fig. 6D), which induces oversegmentation (Fig. 6E). However, in the proposed method, which combines GWDT and cell skeleton, the oversegmentation can be avoided. The mesh plot of the GWDT of the mask area for the cell is shown in Figure 6(F). Within the mask area, only one peak is detected. Accordingly, the result shows that there is no local minimum for the grey-weighted distance transform value along the skeleton (Fig. 6G). Therefore, the cell can be successfully detected (Fig. $6 \mathrm{H}$ ).

After the preliminarily segmented mask areas were separated with the proposed method, the contours of the separated subareas are first extracted, as shown in Figure 7(A). To get the optimised cell boundary detection, the contour expansion method was applied to the contours, as what we did previously (Wang et al., 2015). In the contour expansion method, the contours are first parameterised. An energy function involving light intensity and geometries of the contours are constructed. The final contour is obtained by iteratively minimising the total energy of the evolving contour. Driven by the field of light intensity, the contour converges towards and will stop at the actual cell boundary. The optimised cell boundary detection is shown in Figure 7(B).

\section{Comparison with other methods}

To validate the proposed method, a comparison of clustered cell segmentation was implemented with several other popular methods, including the watershed method (Meyer, 1994), an intensity-based method (Wang et al., 2015), a markercontrolled watershed method (Song et al., 2015), and the proposed method with 393 clustered cells. The result is shown in Table 1.

Among the four methods, the proposed method achieves $85.8 \%$ accuracy rate, followed by the intensity-based method, which gives $80.2 \%$ accuracy rate. The other two methods provide a lower accuracy rate of around $70 \%$. Authors want to note here that all cells involved in the evaluation in this study (Table 1 and whole cell evaluation in Fig. 9) were manually confirmed one by one. Because all the sample images were captured using time lapse phase contrast microscope with a certain time interval, all cells can be manually distinguished with the assistance of their adjacent frames. This guarantees the validity of the ground truth selected for evaluation. 


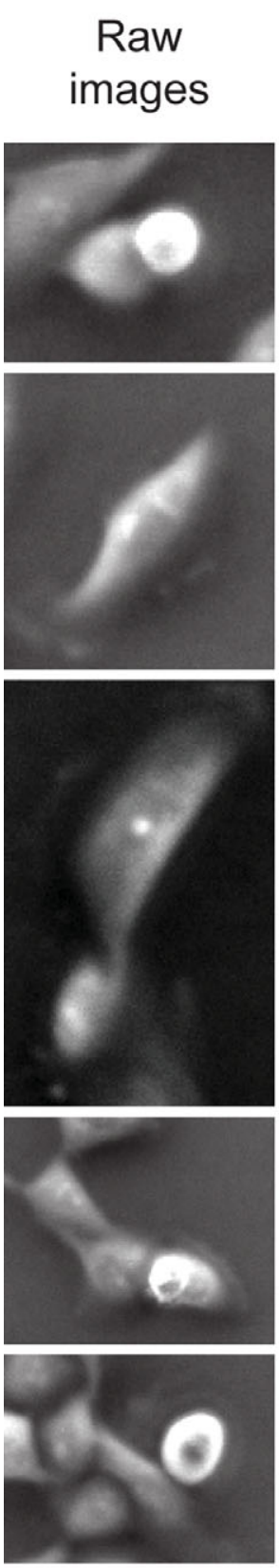

(A)
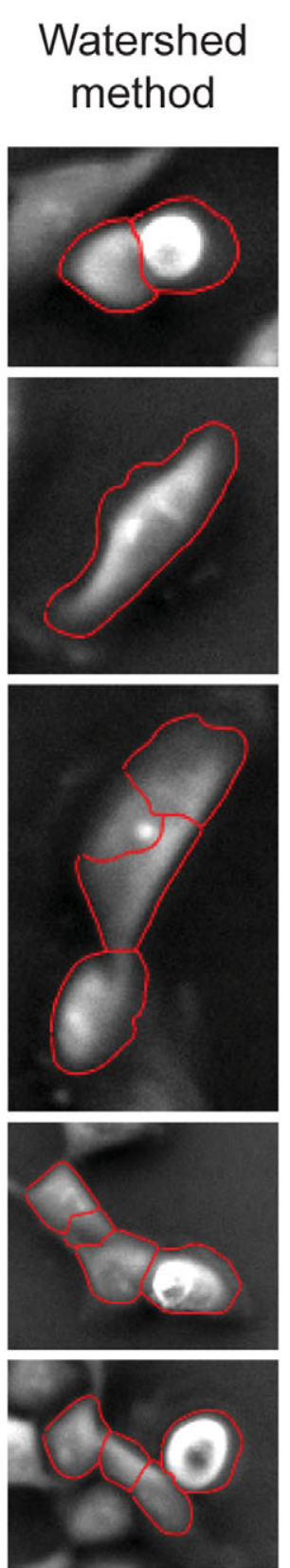

(B)
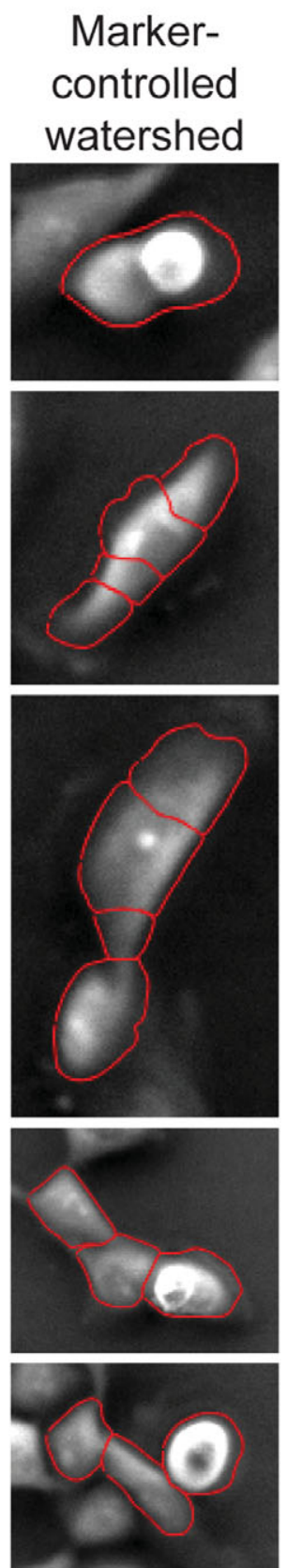

(C)
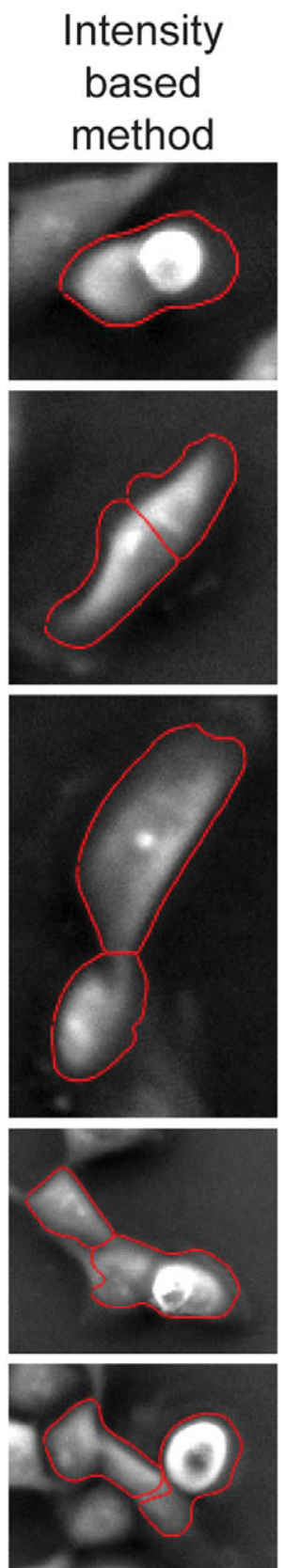

(D)
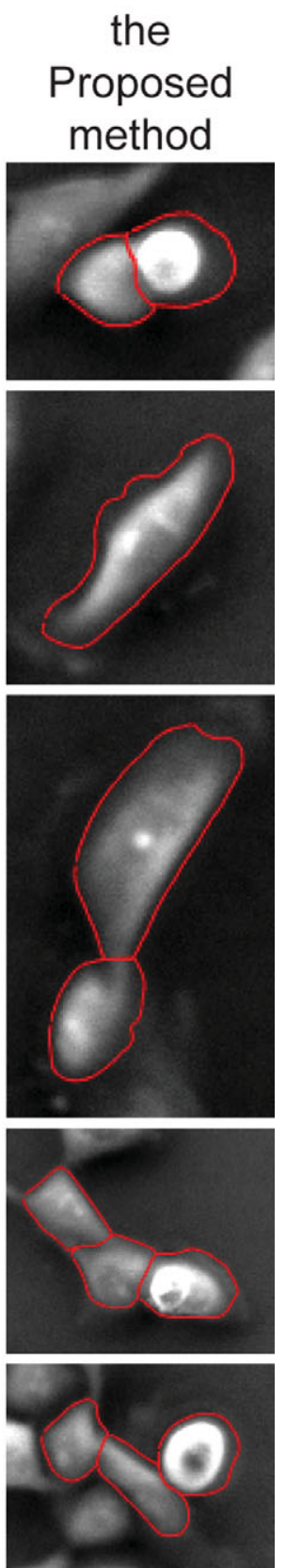

(E)

Fig. 8. Comparison of cell segmentation with different methods. (A) Raw negative phase contrast images of candidate clustered cells. (B)-(E) Segmentation results by watershed method (Meyer, 1994), marker-controlled watershed method (Song et al., 2015), intensity-based method (Wang et al., 2015) and the proposed method, respectively.

Compared with the other three methods, especially the watershed method and the shape-based method, the proposed method has much lower oversegmentation rate. Figure 8 shows the comparison of some typical examples of clustered cell separation. The first column shows the raw images of typical clustered cells, including mitotic cells, elongated cells and clustered cells.
Among the four methods, the watershed method (Meyer, 1994) and the marker-controlled watershed method (Song et al., 2015) can easily induce oversegmentation. Both methods are shape-based methods. The light intensity information within the mask areas is ignored. This will cause oversegmentation, especially when it comes to the mitotic cells which normally have much higher image contrast. The light 


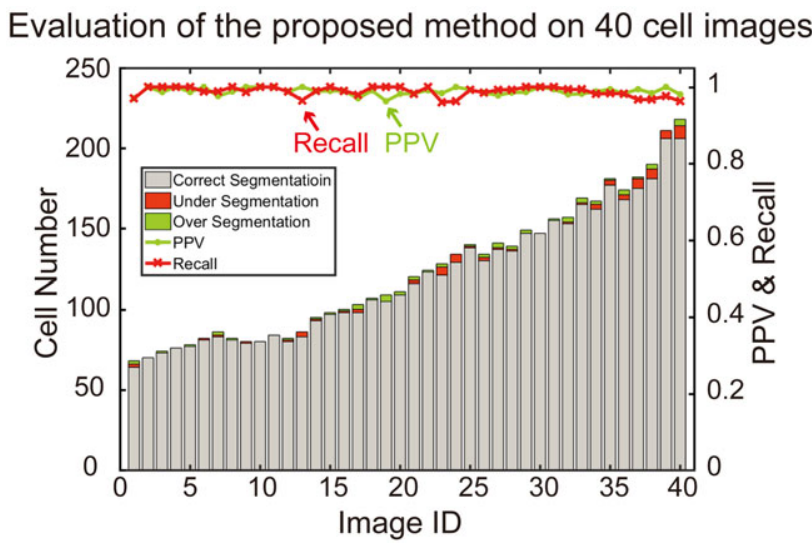

Fig. 9. Whole cell evaluation of the proposed method. The stacked bars show cell number of correct segmentation, under segmentation and over segmentation, scaled by the left vertical axis. The line plots show the PPV and recall, scaled by the right vertical axis.

intensity-based method (Wang et al., 2015) took only the light intensity information into account. When there are multiple pseudo-peaks, especially to elongated cells, the method will cause oversegmentation. The proposed method integrates both light intensity information and the shapeinformation and achieves the improved segmentation results, as shown in the fifth column in Figure 8.

\section{Whole image evaluation}

The proposed method was implemented on the whole image of 40 negative phase contrast images. Undersegmentation, over- segmentation, corresponding positive predictive value (PPV) and recall were shown in Figure 9. The overall accuracy rate, PPV and recall of cell segmentation using the proposed method are $97.16 \%, 98.82 \%$ and $98.64 \%$, respectively.

The image segmentation of four negative phase contrast images is demonstrated in Figure 10. For four raw phase contrast images shown in Figure 10(A), corresponding segmentation results are given in Figure 10(B). Under segmentation and over segmentation are marked by yellow and green arrows, respectively.

The accuracy rate of the algorithm is limited by the initial segmentation of binarisation and the skeleton-based separation method. The performance of adaptive binarisation in the initial segmentation needs to be improved, especially when cell density is high. A possible way to further improve the accuracy rate of the clustered cells could be the application of the deep learning or machine learning methods.

\section{Conclusion}

In summary, we have developed a new method for clustered cell segmentation in negative phase contrast images of MCF 10A human breast cells. This is done by fusing cell shape and light intensity information through the construction of greyweighted distance transform (GWDT). The method consists of two steps. In the first step, the clustered cells are detected by peak detection in GWDT. This avoids the pseudo-peaks induced by intercellular organelles and reversed image contrast for cells with large height. The second step is the separation of
(A)

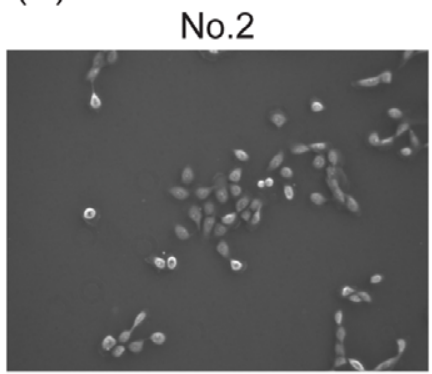

(B)

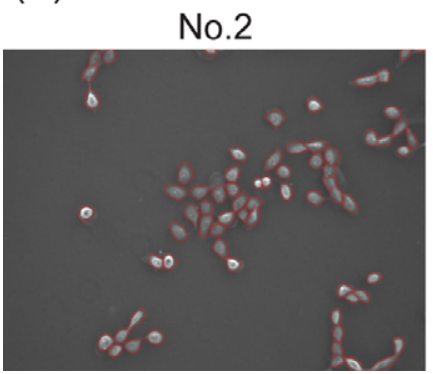

Raw images
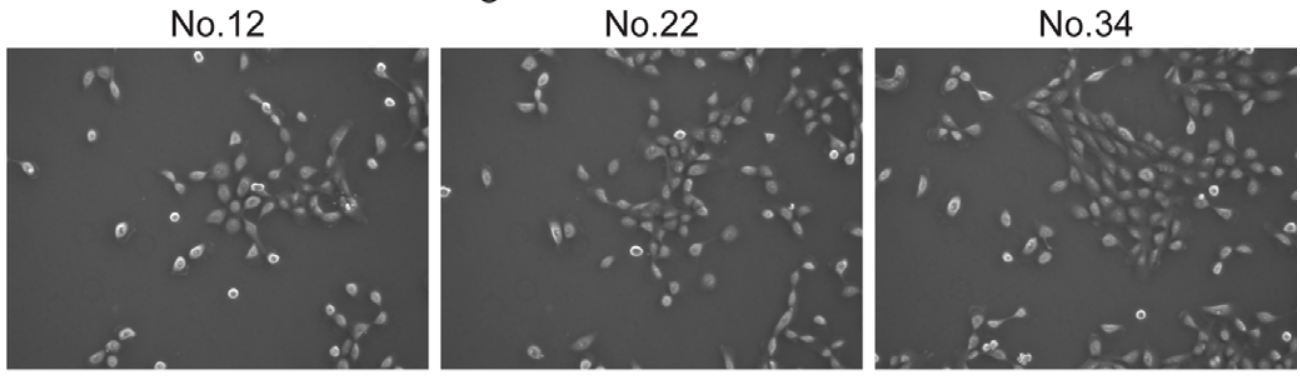

Fig. 10. Cells segmentation results on whole cell images. (A) Raw negative phase contrast images of MCF 10A cells. (B) Segmentation results with the proposed method. Under segmentation and over segmentation are pointed by yellow and green arrows, respectively. 
clustered cells. To do this, a modified region skeleton method was proposed. The value of GWDT along the detect skeletons was used to separate clustered cells. Compared to the widely applied shaped-based or light intensity-based methods, the proposed method provides more accurate detection and hence better separation of clustered cells. The achieved accuracy rate is $85.8 \%$ and $97.16 \%$ for clustered cells and all cells, respectively, in negative phase contrast images.

\section{Acknowledgements}

This study is financially supported by National Natural Science Foundation of China (Grant No. 51775028) and Beijing Natural Science Foundation (Grant No. L150002).

\section{References}

Al-Kofahi, Y., Lassoued, W., Lee, W. \& Roysam, B. (2010) Improved automatic detection and segmentation of cell nuclei in histopathology images. IEEE Trans. Biomed. Eng. 57, 841-852.

Ali, R., Gooding, M., Christlieb, M., Brady, M. \& Ieee (2008) Advanced phase-based segmentation of multiple cells from brightfield microscopy images. In Proceedings of the 2008 5th IEEE International Symposium on Biomedical Imaging: From Nano to Macro. pp. 181-184. IEEE, Paris, France.

Ali, S. \& Madabhushi, A. (2012) An integrated region-, boundary-, shapebased active contour for multiple object overlap resolution in histological imagery. IEEE Trans. Med. Imaging 31, 1448-1460.

Bai, X. \& Latecki, L.J. (2008) Path similarity skeleton graph matching. IEEE Trans. Pattern Anal. Mach. Intell. 30, 1282-1292.

Chalfoun, J., Kociolek, M., Dima, A., Halter, M., Cardone, A., Peskin, A., Bajcsy, P. \& Brady, M. (2013) Segmenting time-lapse phase contrast images of adjacent NIH 3T3 cells. J. Microsc. 249, 41-52.

Chen, X., Zhu, Y.Q., Li, F.H., Zheng, Z.Y., Chang, E.C., Ma, J.W. \& Wong, S.T.C. (2015) Accurate segmentation of touching cells in multi-channel microscopy images with geodesic distance based clustering. Neurocomputing 149, 39-47.

Chenyang, X. \& Prince, J.L. (1998) Snakes, shapes, and gradient vector flow. IEEE Trans. Image Process 7, 359-369.

Chin, R.T., Wan, H.-K., Stover, D.L. \& Iverson, R.D. (1987) A one-pass thinning algorithm and its parallel implementation. Comput. Vision, Graph., Image Process. 40, 30-40.

Chowdhury, S., Kandhavelu, M., Yli-Harja, O. \& Ribeiro, A.S. (2013) Cell segmentation by multi-resolution analysis and maximum likelihood estimation (MAMLE). BMC Bioinform. 14, S8-1-S8-13.

Cosatto, E., Miller, M., Graf, H.P., Meyer, J.S. \& IEEE (2008) Grading Nuclear Pleomorphism on Histological Micrographs. In Proceedings of the 19th International Conference on Pattern Recognition, Vols 1-6. IEEE, New York.

Das, D.K., Mukherjee, R. \& Chakraborty, C. (2015) Computational microscopic imaging for malaria parasite detection: a systematic review. J. Microsc. 260, 1-19.

Dewan, M.A.A., Ahmad, M.O. \& Swamy, M.N.S. (2014) A method for automatic segmentation of nuclei in phase-contrast images based on intensity, convexity and texture. IEEE Trans. Biomed. Circuits Syst. 8, 716-728.
Ersoy, I., Bunyak, F., Palaniappan, K., Sun, M. \& Forgacs, G. (2008) Cell spreading analysis with directed edge profile-guided level set active contours. In Proceedings of the International Conference on Medical Image Computing and Computer-Assisted Intervention, Part I. Vol. 11, pp. 376383. Springer, Berlin, Heidelberg.

Gharipour, A. \& Liew, A.W.C. (2016) Segmentation of cell nuclei in fluorescence microscopy images: an integrated framework using level set segmentation and touching-cell splitting. Pattern Recog. 58, $1-11$.

Holt, C M., Stewart, A., Clint, M. \& Perrott, R.H. (1987) An improved parallel thinning algorithm. Commun. ACM 30, 156-160.

Irshad, H., Veillard, A., Roux, L. \& Racoceanu, D. (2014) Methods for nuclei detection, segmentation, and classification in digital histopathology: a review-current status and future potential. IEEE Rev. Biomed. Eng. 7, 97-114.

Kim, K.B., Lee, S.G. \& Kim, G.H. (2014) Nucleus extraction of uterine cervical pap-smears using marker information and watershed algorithm. J. Med. Imag. Health Inform. 4, 1-7.

Kong, H., Gurcan, M. \& Belkacem-Boussaid, K. (2011) Partitioning histopathological images: an integrated framework for supervised color-texture segmentation and cell splitting. IEEE Trans. Med. Imaging 30, 1661-1677.

Kumar, S., Ong, S.H., Ranganath, S., Ong, T.C. \& Chew, F.T. (2006) A rule-based approach for robust clump splitting. Pattern Recog. 39, 1088-1098.

Lin, G., Adiga, U., Olson, K., Guzowski, J.F., Barnes, C.A. \& Roysam, B. (2003) A hybrid 3D watershed algorithm incorporating gradient cues and object models for automatic segmentation of nuclei in confocal image stacks. Cytometr. Part A 56A, 23-36.

Meyer, F. (1994) Topographic distance and watershed lines. Signal Process. 38, 113-125.

Piccinini, F., Bevilacqua, A. \& Lucarelli, E. (2013) Automated image mosaics by non-automated light microscopes: the MicroMos software tool. J. Microsc. 252, 226-250.

Roth, D.B. \& Gerhard (2007) Adaptive thresholding using the integral image. J. Graph. GPU Game Tools 12, 13-21.

Seroussi, I., Veikherman, D., Ofer, N., Yehudai-Resheff, S. \& Keren, K. (2012) Segmentation and tracking of live cells in phase-contrast images using directional gradient vector flow for snakes. J. Microsc. 247, 137146.

Serrat, J., Lopez, A. \& Lloret, D. (2000) On ridges and valleys. In Proceedings of the 15th International Conference on Pattern Recognition. ICPR-2000. Vol. 4, pp. 59-66. IEEE, Barcelona, Spain.

Soille, P. (1994) Generalized geodesy via geodesic time. Pattern Recognit. Lett. 15, 1235-1240.

Song, Y.Y., Zhang, L., Chen, S.P., Ni, D., Lei, B.Y. \& Wang, T.F. (2015) Accurate segmentation of cervical cytoplasm and nuclei based on multiscale convolutional network and graph partitioning. IEEE Trans. Biomed. Eng. 62, 2421-2433.

Telea, A. \& Van Wijk, J.J. (2002) An augmented fast marching method for computing skeletons and centerlines. In Vissym Proceedings of the Symposium on Data Visualisation, pp. 251-259. Eurographics Association, Barcelona, Spain.

Veta, M., van Diest, P.J., Kornegoor, R., Huisman, A., Viergever, M.A. \& Pluim, J.P.W. (2013) Automatic nuclei segmentation in H\&E stained breast cancer histopathology images. PLoS One 8, e70221.

Vink, J.P., Van Leeuwen, M.B., Van Deurzen, C.H M. \& De Haan, G. (2013) Efficient nucleus detector in histopathology images. J. Microsc. 249, 124-135. 
Wahlby, C., Sintorn, I M., Erlandsson, F., Borgefors, G. \& Bengtsson, E. (2004) Combining intensity, edge and shape information for 2D and 3D segmentation of cell nuclei in tissue sections. J. Microsc. 215, 67-76.

Wang, Y., Jeong, Y., Jhiang, S M., Yu, L. \& Menq, C.-H. (2014) Quantitative characterization of cell behaviors through cell cycle progression via automated cell tracking. PLoS One 9, e98762.

Wang, Y., Zhang, Z., Wang, H. \& Bi, S. (2015) Segmentation of the clustered cells with optimized boundary detection in negative phase contrast images. PLoS One 10, e0130178.
Wienert, S., Heim, D., Saeger, K. et al. (2012) Detection and segmentation of cell nuclei in virtual microscopy images: a minimum-model approach. Sci. Rep. 2, 503-1-503-7.

Xing, F. \& Yang, L. (2016) Robust nucleus/cell detection and segmentation in digital pathology and microscopy images: a comprehensive review. IEEE Rev. Biomed. Eng. 9, 234-263.

Yi, F., Moon, I., Javidi, B., Boss, D. \& Marquet, P. (2013) Automated segmentation of multiple red blood cells with digital holographic microscopy. BIOMEDO 18, 026006-026006.

Zhang, T.Y. \& Suen, C.Y. (1984) A fast parallel algorithm for thinning digital patterns. Commun. ACM 27, 236-239. 